\title{
EFFICIENT FLYBACK CONVERTER WITH H6 INVERTER USING MATHEMATICAL MODELLING OF SMFO FOR PERFORMANCE IMPROVEMENT IN GRID CONNECTED PV SYSTEM
}

\author{
Saravanakumar S And Arulmozhiyal R \\ sskresearch2016@gmail.com \\ ${ }^{1}$ Assistant Professor, Department of Electrical and Electronics Engineering, N.S.N College of Engineering and \\ Technology, Karur. \\ ${ }^{2}$ Professors, Department of Electrical and Electronics Engineering, Sona College of Technology, Salem, India.
}

\begin{abstract}
The proposed flyback inverter controlled by Stochastic Moth Flame Optimization-based maximum power point tracking (MPPT) converter for the small photovoltaic (PV) power system. The system of small PV could be connected easily in a parallel manner for yielding higher output power. Once a power system PV is being constructed with the small power system, the cost of entire system will enhance and this will be matter of concern. So as to overcome this issue, this approach presents a PV system which in turn employs no expensive DC sensor however employs the technique for the estimation of PV current from PV voltage source. The foremost intention of using mathematical formulation based stochastic moth flame optimization is to boost and optimize the PV input. The optimized source input may be helpful for varying the duty cycle based on maximum power point tracking, at which the optimal range of duty cycle is optimized at each iteration and the optimal range will be taken for pulse generation. Also, the fly back converter is one such DC-DC converter having high level of frequency and is employed in low power application. Due to this high-frequency function, losses and stresses of switching will be more. For reducing the losses and stresses of the interleaved flyback converter is presented. Therefore, the presented system is better for dynamic system application. In the grid-connected system, the leakage current is one of the major shortcomings and to evade this limitation, H6 inverter type is employed. By this H6 inverter advantage and fly back converter benefit, this approach mainly concentrates on the stresses over the switches and thus eradicating the leakage current and thereby reducing the Harmonics level.
\end{abstract}

Keywords: Flyback inverter, Mathematical modeling, Stochastic Moth Flame Optimization based maximum power point tracking, H6 type inverter

\section{INTRODUCTION}

PV energy has been extensively used in numerous manufacturing sectors due to the growing emphasis on renewable energy sources. In centralized, string and ac module systems, the PV power systems are classified. Among them, a grid-connected low-power inverter, termed the microinverter, is mounted on the single PV- module in an ac module system, which records the individual maximum power-point (MPP). In comparison, the AC module system is more stable and simpler to maintain than other PV systems. The ac module PV system was thus recently considered a trend among potential photovoltaic systems with these advantages. With its performance, the type of power, power density, reliability and cost, the value of the microinverter is evaluated. To satisfy these criteria, because of its clear layout circuit and reliability capacity and high efficiency, a topology of flyback inverter was adopted. Besides, both step-up and step-down functions are given in the H6 inverter flyback inverter; this functionality is ideal for PV applications in which the Inverter can run within a broad power range. The flyback inverters might be categorized as continuous current (CCM) and discontinuous current (DCM) mode when they are in constant

Received MAY 18, 2021

www.dynamicpublishers.com;

www.dynamicpublishers.org;
ISSN1056-2176(Print); ISSN 2693-5295 (online)

$\$ 15.00$ CDynamic Publishers, Inc.

https://doi.org/10.46719/dsa202130.10.02 
switching frequency. In reality, the PV inverter that is called a flyback inverter consist of both modes; it eventually works in DCM low instantaneous power or low solar irradiation, while the rated average power in all instantaneous CCMs. The flyback inverter may then be assumed to have a hybrid operating mode for the entire activity period. The flyback has various merits, compared to a flyback inverter with DCM alone, including a higher performance with less current tension, improved strength and a simpler configuration. However, the flyback inverter power transmitting feature of input-to-output current in CCM s having a right semiconductor zero, leading to restrictions on system gain and bandwidth of the controller. As the point of PV operation applications greatly varies, the controller in particular, should protect the minimum RHP zero. The relative gain was planned to be small relatively when a traditional flyback control is added to with H6 Inverter to provide stability in any operating stage. There is essentially very low device gain for the DCM region's fly back inverter. The high gain feedback controller is essential in the operation of DCM for attaining fast tracking reference and the efficiency in the refusals that were distributed. Moreover, the control gain in CCM is being constrained through RHP zero once the standard fly back controller is being added. As a consequence, the low control output in DCM produces inappropriate power efficiency and high overall harmonic distortion (THD). This is why there are also drawbacks to the use of the flyback converter.

In this approach, the strategy of current control in terms of flyback inverter with H6 Inverter is presented. By applying the Stochastic Moth Flame Optimization based MPPT with Stochastic Moth Flame Optimization based MPPT offers the performance of elevated tracking and improved interruption elimination in both modes of operation and in turn strengthen several flyback inverter advantages. The remaining section of the paper can be structured as follows: In Section II, the related existing methodology can be reviewed. Section III represents the suggested methodology and in turn manages huge issues on the traditional current control strategies. After that,m the presented control strategy is being introduced with their validity and superiority from the theoretical point of analysis. At last, the experimental and simulation outcomes are signified in section IV.

\section{RELATED WORKS}

[1] In which the photovoltaic ( PV ) energy pumped into three-phase grid is optimized by the two controllers. The first controller is used to precise the DC voltage that can be used by the 3-phase Inverter in unbalanced climatic conditions to monitor the optimum power point of a photovoltaic generator. A polynomial interpolation based on the principle of Lagrange is used for this new controller. A robust sliding mode control (SMC) is a core element of the second control. It helps to monitor the active and reactive power injected into the network so that high reliability and absolute minimal harmonic distortion can reach full active grid power using the same three-phase Inverter. [2] this research is aimed at researching recent integration criteria including voltage sag, flicker voltage, harmonics, unbalance of voltage and variance in frequency. Compliance controls and compliance procedures are also developed to satisfy these criteria. A large 3-phase PVPP connected to the grid is built in line with these specifications. An updated inverter controller is designed to alleviate the sage occurrence without any external system and to meet the low voltage driving requirement. It can work effectively, and when sags and errors are observed, the mode of operation can be modified, and a reactive current dependent on a sag depth can be injected. The voltage controller and dynamic voltage regulator are intended to regulate the tension fluctuation and unbalance between the PVPP and the grid at the point where it is normal to connect it. The voltage and current are minimized by 
means of a proper design and an RLC filter below the defined limits. [3] Provides the suggested control scheme for the grid-connected PV system under the increasingly variating solar radiation levels, together with the novel optimum operating pattern. All PV panel in the suggested configuration cascade a step-up boost converter and thus enables independent power corresponding to degrees of irradiation, generating several levels of dc-voltage and being converted to ac by means of the active neutral point clamped Inverter (ANPC). For each boost converter unit, an altered MPPT-based salpole swarm algorithm (DWSSA) has been proposed that enables convergence and iteration stability effectively to be accelerated to quickly change the quest mechanism to various fast variable amounts of solar irradiation.[4] presented a control strategy for enhancing the dynamics of PV device where PV is being controlled with the use of fractional order PID controller that turns into several regions with the use of fractional order PID controller and the patterns of partial shading along with particle swarm optimization, wheras PV current controlled with the use of sliding mode controller that didn't requires pulse width modulation (PWM).[5] proposes a new MPPT based on Metaheuristic approach for Manta Ray Food Optimization (MRFO). The MRFO-based MPPT proposed is intended to derive from the shadow-operated solar array Triple-Junction the Global Peak Power Point (GMPP). Seven shadow variations on seven topologies of three solar arrays are studied. [6] To overcome the MPPT issue for the PV energy system, a new hybrid moth flameoptimizer-perturb and observing process called HMFOPO is used to provide an optimum solution with the full capacity of the PV modules. The suggested scheme explores the photovoltaic curve and applies the $\mathrm{P} \& \mathrm{O}$ approach from the best slogan of the MFO algorithm by using the MFO optimization method. [7] enter into an exhaustive analysis of state-of-the-art photovoltaic (PV) systems with a partial shading condition (PSC) for Optimum PowerPoint Monitoring (MPPT). The use of different MPPT control approaches is especially important in order to guarantee the highest effective, efficient extraction of power in PV systems.[8] focus on the optimum configuration of a renewable energy system (RES) based on the grid and off-grid mix. Photovoltaic (PV), Wind Turbine (WT) and Fuel Cell (FC) are the components of this device with gaseous hydrogen tank for the chemical storage of the electricity. Using a new meta-heuristic optimization strategy, the optimum size of the proposed hybrid generation system is obtained. This optimization technique is intended to boost the efficiency of the traditional Artificial Ecosystem Optimization (AEO) algorithm, called Enhanced Artificial Ecosystem Optimization (EAEO). [9] the proposal of a new approach for optimizing photovoltaic systems in real-time adaptive reactive energy depends on the enhanced algorithm of optimization for dynamic learning and teaching. The process of learning is streamlined continuously through the mathematic model building for reactive voltage regulation in a photovoltaic power station. The strategy of optimum collaboration among the AVC/RPVC control system and the intelligent power grid control was well-defined for the real-time data, and the real time system, dynamic and adaptive control strategy is applied. [10] This paper discusses an early study of the active power limit, volt watt laws, smart PV inverter frequency watt change and the effects on the voltage and the frequency. [11] suggested a real-time SG communication device.[12] presented an efficient technique for employing a key and clear variable model of research so as to track structures of PV. [13] Presents the flyback inverter for the small photovoltaic (PV) power system operated through sensorless current MPPT. Whilst the suggested device has a limited output capacity of $300 \mathrm{~W}$. It is possible to conveniently connect a few small PV power systems sets for enhancing the power of output. If a system of PV is installed with a variety of a small power systems, the overall costs of the system will rise and are a problem. [14] a new control technique is suggested here, with the phased photovoltaic grid-connected inverter topology. In the interleaved flyback, the 
Inverter is constant and vital, the single flyback and the single reverse. The sporadic alternating operating mode alternates between jobs. The control strategy is sufficient for each inverter power level, and it can also increase the reliability, and the output of the grid linked current of the Inverter at each level.

\section{PROBLEM STATEMENT}

The use of nonlinear loads on end-users of electricity is typically a source of harmonic distortions. Nonlinear loads, most of which are charged with electronic power supplies, produce a power output that is non-sinusoidal. There is a need for an optimized system to maximize THD output in Grid-connected PV system in order to eliminate harmonics and to switch noise.

\section{PROPOSED METHODOLOGY}

This section is the deliberation of a thorough explanation of the proposed scheme. The flow of the proposed system, on the whole, is shown below in figure 1:

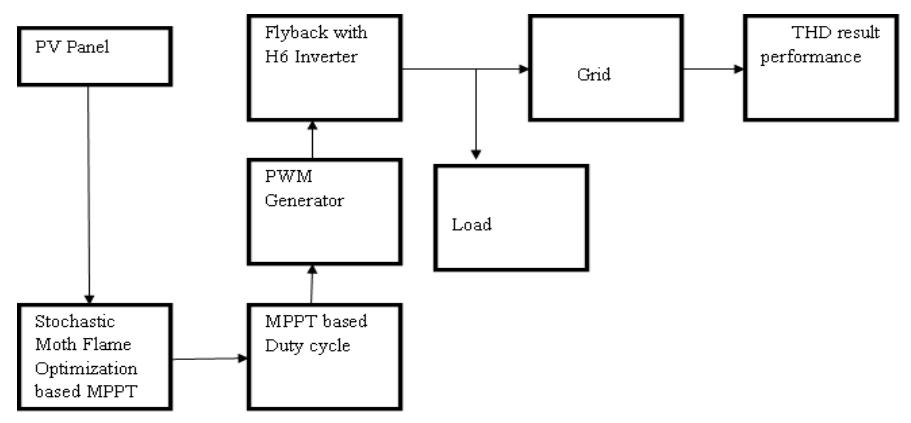

Figure 1 suggested method graphic illustration

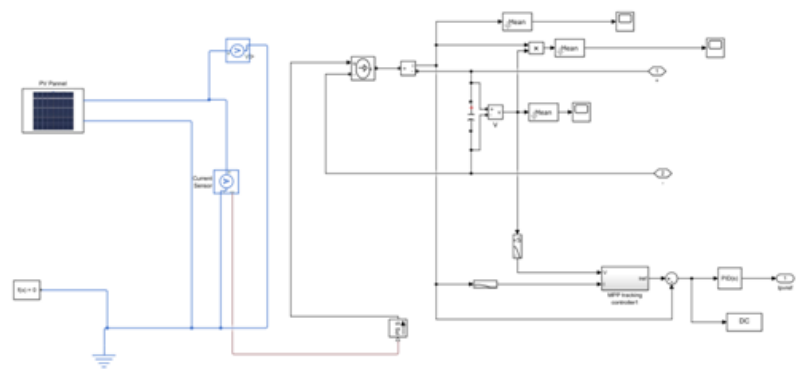

Figure 2 PV panel Design

The design of a PV panel, as depicted in figure 2. At this time the numerical model that explains the behavior dynamically. In last few decades, the wide-ranging arithmetical of solar cells model were researched. From this consideration the PV plant model concentrate in the intricate model of PV build and reviewed in previous study. A PV cell in turn absorbs the energy in anticipation of illuminating the photons. A central portion is the junction of P-N for cells PV which could take up radiation from solar. This in turn happen as a light that strikes the invalid stuff. The quantity saved 
directly overtakes the device as a load in solar cell is being incorporated to this before the radiance cease. The Equation reveals the fact that the supplied current from solar cell in series resistance $R_{S}$ is low relatively, furthermore shunt $R_{s} h$ was comparatively strong. The representation comprises of a supply of current, certain résistance, and a diode. The entire resistors were not considered for simulation enhancement. The covers the present source was covered by a diode, equivalent mode and circuit for PV cells resistors in parallel/series. The mathematical equations of the current output (out) depending on the abbreviation are employed. The current of the PV cell is signified by the following model of equation,

$v^{\text {out }}=v^{\text {Photon }}-v^{R S}\left[\left(e^{-t i \theta Q\left(I^{\text {out }}\right.}+R_{s r} * I^{\text {out }}\right)-1 / R_{p r]} \sigma q c-1\right)-1 / R_{p r}\left(I^{\text {out }}+R_{s r}\left(I^{\text {out }}+R_{p r} * v^{R S}\right)\right.$

Sustainable operation conditions of the system of hybrid power intended on behalf of using zero reactive power, a magnet synchronous generator was employed. Current and Voltage attained are mathematically expressed,

$V_{R E}=3 / 5 / \pi v_{\text {stator }}$

$V_{R E}=\pi / \sqrt{1} / \pi v_{\text {stator }}$

The PV system expected output is,

$E^{\text {out }}=\operatorname{Ig}_{\mathrm{c}} C_{R_{s}}-F C_{d}+q C_{R_{s} k h}$

where $\mathrm{q}$ is a $1.6 \times 10^{-19} \mathrm{C}$ electric charge, $\mathrm{K}$ refers to the Boltzmann's constant, the idealizing cell factor is $F, I_{g c}$ is the light generated current, the $c_{d}$ is the voltage of diode, and cell's absolute temperature is $\mathrm{T}_{\mathrm{c}}$.

\section{Stochastic Moth Flame Optimization based MPPT}

It is a meta heuristic algorithm imitating moth mitigation in the evening. The foremost intention of using stochastic moth flame optimization is to boost and optimize the PV input. The optimized source input may be helpful for varying the duty cycle based on maximum power point tracking, at which the optimal range of duty cycle is taken automatically for pulse generation. Whereas, in case of not using optimization technique, the duty cycle is varied and we have to choose the best duty cycle range, but in this the duty cycle is optimized at each iteration process in training process. On varying the duty cycle in an optimized manner, the pulse generation will be better. Moths exploit particular navigation techniques, named as TO (transverse orientation), to fly in night through keeping a rigid angle with regard to the moon. In other terms, TO navigation method is simply competent for straight line flying while the light source is extremely far away. As the moths are being tricked by artificial human-made light, they fly around the light spirally. Actually, they attempt in straight line to keep a similar angle to fly with that of the light. As such a light is extremely close on comparing moon, though; keeping a related light source angle to cause a spiral fly path for moths deadly. In this, the moth converges towards the light eventually and these activities were mathematically modeled to suggest MFO (moth-flame optimization) algorithm.

In the MFO framework, there were two key mechanisms: moths $(\mathrm{X})$ and flames $(\mathrm{F})$. These are solutions of the optimization problem and the distinction among them set in their updating technique utilized all through the process of optimization. The moths are actually the search agents that are in motion in the search space region, while flames are the unsurpassed moth's position which attains 
thus far. Hence, all moths search something like a flame and update it in finding an enhanced solution. With this method, a moth not at all loses its best solution.

The present study signifies that the typical MFO is not capable of tackling the steel frame structures optimization problem efficiently with distinct mean variables and the process converge to the local optima. In this approach, a modified version of this algorithm termed as stochastic moth-flame (SMFO) meta-heuristic algorithm is proposed in order to enhance the MFO to acquire more consistent solutions. Since the primary alteration, the best information derived by the swarm throughout the preceding iterations, is involved efficiently in the proposed SMFO framework for generating a new swarm.

The moth is the candidate solution, and in this algorithm, the moth locations are the problem parameter. Motors may then switch to 1-D, 2-D, 3-D, or hyperdimensional space with modified position vectors. The algorithm SMFO is a populbation-based algorithm. As a population-based algorithm, $\mathrm{n}$ moths are used as search agents in the problem field. Flames have been the best moth positions ever attained. The moth then scans for a flag and changes it if there is a better alternative. Thus, fires often constitute spatial data points.

The use of stochastic process refers to the formulation of optimization technique in a mathematical manner. Thus, the mathematical formulation of the optimization process is employed at which the random variables are employed for mathematical functions. The stochastic process is the kind of system that evolves in time on undergoing the fluctuation chances. This system can be described by defining the random variables. Thus, the stotachastic behavior based moth flame optimization formulation is given below:

$\mathrm{M}\left(P_{i}, K_{j}\right)=E_{i}, e^{b t} \cdot \operatorname{Cos}(2 \pi \mathrm{t})+K_{j}$,

Where $E_{\mathrm{i}}$, is the euclidian distance of the ith moth for the

$\mathrm{j}$ th flame, $t$ is the random number, $\mathrm{P}$ is the moth, and $\mathrm{K}$ is the flame.

As can be seen in the above formula, the next position of a moth is defined for a burn. In the spiral system, the t element determines how close the next moth is to burn. The fire will, therefore, infer the hyper ellipse in all directions, and the following position of the moth will be put in this space. In comparison to their fires, this approach tends to allow more effective use of moths. A moth has to change its location only with a single burn, not only to raise the possibility of becoming a global solution. In each iteration and after flames modifying, the flames were categorized as per their fitness values.

$$
M_{\text {flames }}=\operatorname{round}(\mathrm{M}-1 *(M-1 / T))
$$

At which, the iteration number is represented by $1, \mathrm{M}$ is the number of flames, and utmost of iteration is represented by T. After that, the best fitness value can be calculated. For each optimization algorithm, the fitness value can be calculated.

$$
\text { Best }=\min _{i=1}^{M} O P_{*}^{i}
$$

Where OP is the optimal solution having an ith iteration number. 
The moth fly optimization algorithm is used to monitor MPPT of a PV device, which includes a number of local MPPs in the P-V characteristics. If series connections are made to PV modules. The $\mathrm{N}$ number of modules is used in the PV list. Each module must also be regulated by voltage. The service cycle can be determined with the G-best value. The pulses for PWM Pulse Width Modulation (PWM) converters can be generated with a two-level topology by the PWM generator block. The pulses are created by contrasting a triangular waveform with a modulating signal of comparison. The secondary switches are powered by a PWM signal in the grid-connected operating mode, determined through the PWM generator of the gridline occurrence. It is possible to produce modulation signals by the PWM generator itself, or they can be related by a vector of external signals at the block input. To produce the pulses, one reference signal is required. The phase of the reference signals modulation (amplitude), frequency, and are set to manage the output voltage (Inverter's terminals of $\mathrm{AC}$ ) that is connected to the PWM Generator block connected.

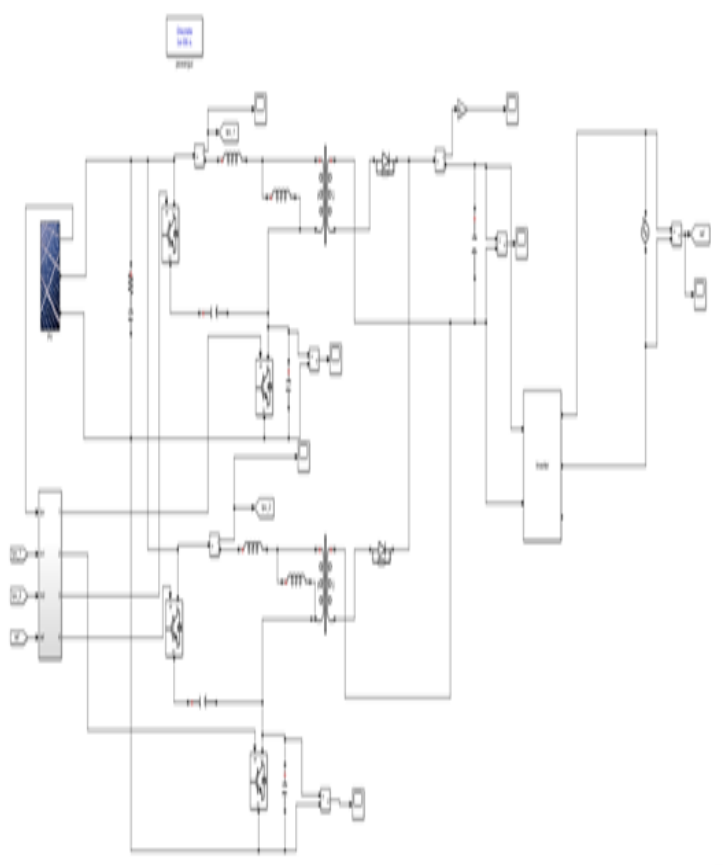

(a) Flyback converter

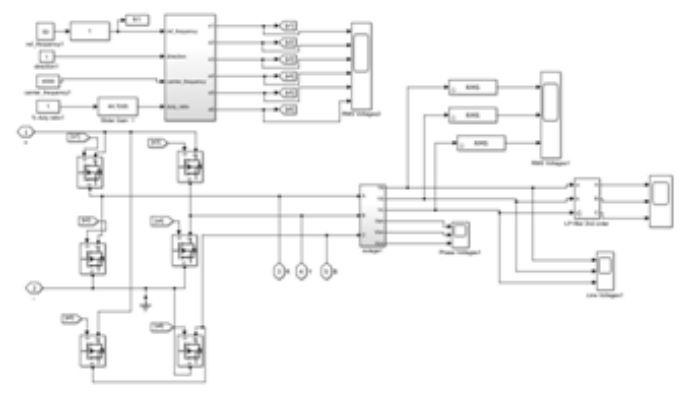

(b) H6 Inverter 


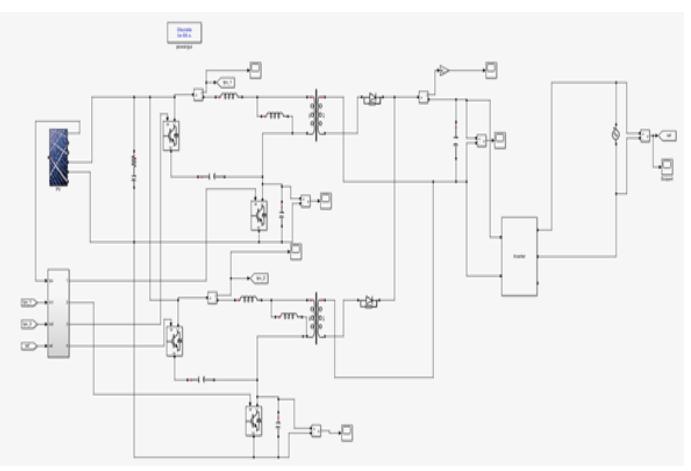

(c) overall design circuits

Figure 3 proposed scheme of a flyback converter with H6 Inverter

Figure 3 represents the overall proposed flyback inverter scheme. In this, the PV source is presently followed by a primary switch, transformer, and switches.

The flyback converter is a topology of power supply, using a mutually coupling inductor to conserve energy when the current is flowing through and releasing energy when power is lost. The energy saved by the magnet field is released after a rapid reversal of the terminal voltage when the current flowing through an inductive circuit is turned off. If a diode is used to handle the saved energy, the diode is called a flyback diode. It just takes a winding on the wire, so the pipe is called a flyback transformer. The fascinating aspect of this arrangement is to transfer electricity only while the main switch is off on the secondary side of the power supply. A relatively limited number of components are used for the simple flyback converter. The input voltage is chopped by a switching system; the primary energy is transmitted by a switching transformer to the secondary one. The secondary diode corrects stress as the condenser smoothes the corrected tension. The supplementary transition between the positive terminal of the PV array and the terminal forms a new current direction. Here you can enter into the H5 inverter topology. This refers to a topology of the H6 Inverter. The topology suggested consists of H6-inverter flyback converter, as shown in Figure 5. The input source for this Inverter is used in the PV module. The output load is connected with the grid line. This Inverter contains a condenser, key switch and diode. The Sp switch key functions on a high level of switching. The residual frequency of switches is close to the frequency of the grid line. The condenser must minimize input voltage and current ripples because the MPPT technique demands approximately uniform input voltage and current. The transformer flyback provides the AC power and isolates the photovoltaic module and the network to avoid accidents. The H6 Inverter decreases the harmonic portion and generates a current waveform in the voltage of the line.

\section{Modes of operation}

\section{Mode I (Interval $0<t<t 1)$ :}

At the switching cycle initiation, the primary switch $S_{l}$ is turned ON. A resultant switch is turned on for the total positive half of the ac cycle, while secondary switches stay ON for a total of the ac 
half negative cycle. In this mode, the input PV voltage is applied to the magnetic induction of the transformer $L_{m}$. Therefore, there is an increase in primary current.

\section{Mode II (Interval $t 1<t<t 2)$ :}

At the initiation of the switching cycle, At the beginning of the interval, switch $S_{1}$ is turned OFF, while the secondary switches remain ON. The drain-source voltage is not able to enhance the high voltage instantly because of the output capacitor. The drain-source voltage increase over the switch $S_{1}$ is delayed due to the capacitor charging. This mode moves towards the end once the capacitor was charged to its highest $\mathrm{V}_{\mathrm{dc}}+\mathrm{Nv}_{\mathrm{ac}}$ value. This alteration of switch $\mathrm{S}_{1}$ from ON state to OFF state occurs in a short period

\section{Mode III (Interval $t 2<t<t 3)$ :}

This mode started when the secondary switches were turned OFF. As the magnetizing inductance, $\mathrm{L}_{\mathrm{m}}$ was charged in the previous interval in the opposite direction, and the current flow in the Inductor at the direction for transferring energy stored in $\mathrm{L}_{\mathrm{m}}$ to the capacitor's input. Accordingly, the primary current $\mathrm{iS}_{1}$ is negative all through this time. The voltage over switch $\mathrm{S}_{1}$ begins to slowly decrease as the capacitor over the switch begins to discharge. This model reaches the end by a complete capacitor-discharge over the switch.

Using the PV current and variable voltage, the MPPT controller measures the PV reference current. In this case, a flyback converter with H6 Inverter is entered as the PV current. Finally, this suggested mechanism is planned to remove the defects by using the Stochastic Moth Flame Optimization using the H6 inverter flyback converter. The distortion and defects would be corrected and stabilized electricity deposited on the grid side.

\section{RESULT AND DISCUSSION}

A $12 \mathrm{~V}$ and $500 \mathrm{~W}$ poly-crystalline solar panel are used as a sustainable energy source for the experimental hardware rig. The production of the solar panel was attached to the H6-inverter MPPT flyback converter. Similarly, the output voltage value of the dc to dc converter that are analogue is attached to controller by the use of the voltage separator. The power transfer from dc to dc is powered by an SG 3525 gate driver from the MOSFET and MPPT controller. The constant PV output supply is connected to a filtered H6 inverter by connecting a source with the AC and LC filter, in order to change the output for H6 Inverter. The MOSFET is chosen as a power switch at the 55amps rating, inverter operation at 100 volts (IRFz44). With gate driver circuit all this MOSFET are connected. By means of MPPT controller the SVPWM is generated and it is offered to the gate driver. At first, $50 \%$ duty cycle is chosen for flyback converter by MPPT controller, after that the feedback output voltage will alter consequently. 
Although $20 \mathrm{Kz}$ switching speed one sample current value is taken for estimating the new duty cycle. Once the current measured is elevated after that the duty cycle is decreased vice versa. Then, the output of flyback converter, the filters and rectifier circuits eradicate the fluctuation. In the H6 block, boosted output 320 volt is inverted into ac at this time; SVPWM and closed loop operation were used. Thus, the H6 inverter pulses are fine tuned for each output cycle. In case, there is a change in output, set value is compared in which the value is different and is then computed as error signal. For H6 the eradicated new PWM is generated.

This process makes sure the correct power switches operation sequence. This method leads in the stabilization of the method across switches, thus soft switching is done here. The output voltage and current has distorted because of switching action. In order to decrease this distortion, the LC filter is connected to output. The Inductor in turn eliminates the current distortion and the capacitor eradicates the voltage distortion. At last, the ac supply is linked with inductive element.

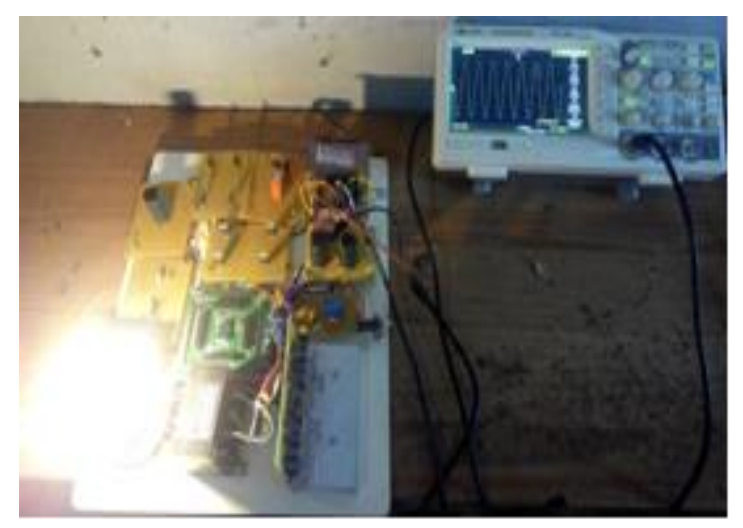

Figure 4 Hardware Prototype Model

The projected method performance analysis is simulated, Various switches were employed and the pulses are evaluated in terms of ON and OFF state condition and the outcomes are illustrated in the graphical form as shown:

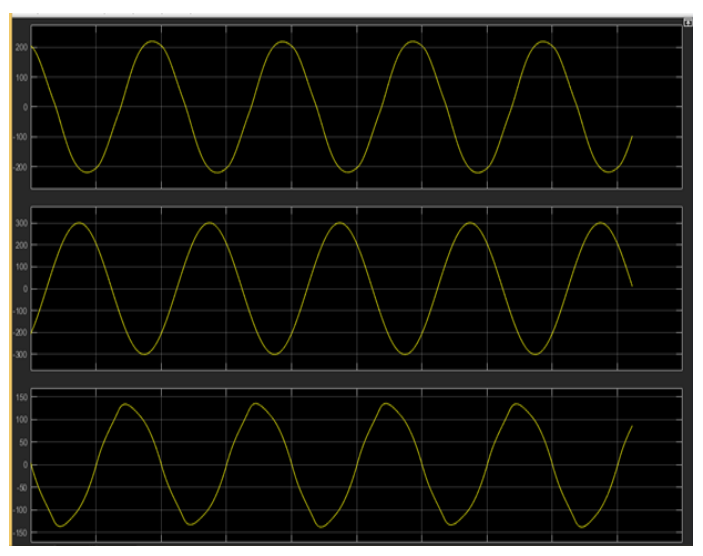

(a) 


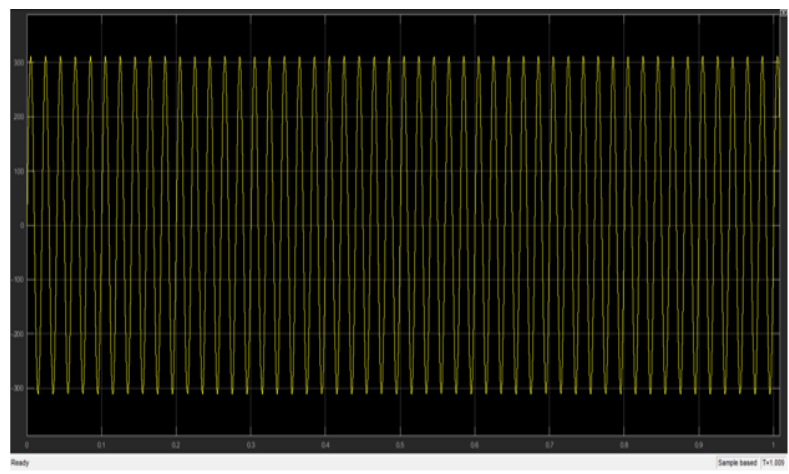

(b)

Figure 5 Inverter output

The experimental waveforms representing the switching operation scheme with their variations were provided in figure 5. The results are evaluated for different time intervals of switching pulses. In this, the voltage and current gate source are estimated and the variation of duty cycle according to the pulse injected is shown in simulation.

\section{a. THD (Total Harmonic distortion):}

Figure 6 illustrated the analysis of THD in the system. From this, the THD is $2.19 \%$ at initialization time 0.06 .

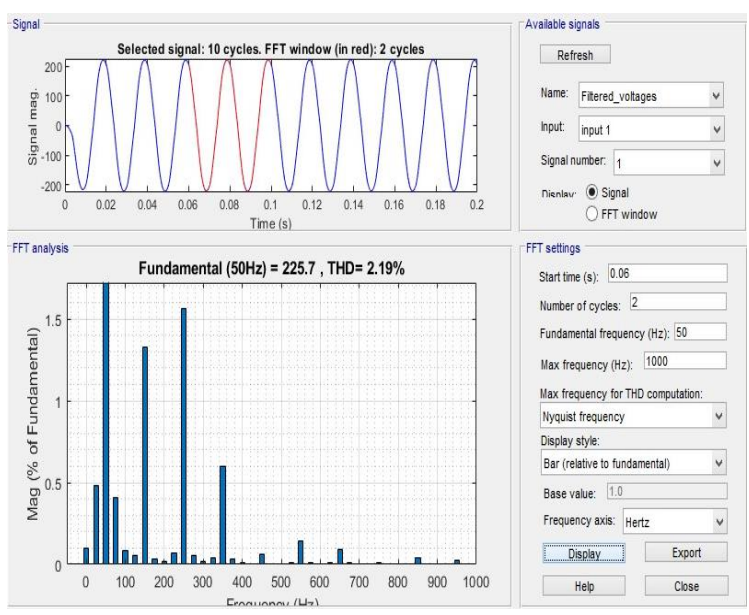

Figure 6 analysis of THD performance with initiation time $0.06 \mathrm{~s}$.

Table 1 is the comparative analysis of the THD values in terms of both proposed and existing [15] techniques.

Table 1 evaluation of THD

\begin{tabular}{|l|l|l|}
\hline Parameters & Existing & Proposed \\
\hline
\end{tabular}




\begin{tabular}{|lr|l|l|}
\hline $\begin{array}{l}\text { Grid } \\
\text { THD (inverter } \\
\text { mode) }\end{array}$ & $3.28 \%$ & $2.19 \%$ \\
\hline
\end{tabular}

The evaluation of the performance of total harmonic distortion is signified and represented in the output waveform shown above. The results depict that the THD value is attained as $2.19 \%$ at the start time of $0.06 \mathrm{~s}$. From the analysis, it was apparent that the proposed system is capable of reducing the total harmonic distortion level, which signifies that the proposed method is better.

\section{b. Efficiency of inverter curve}

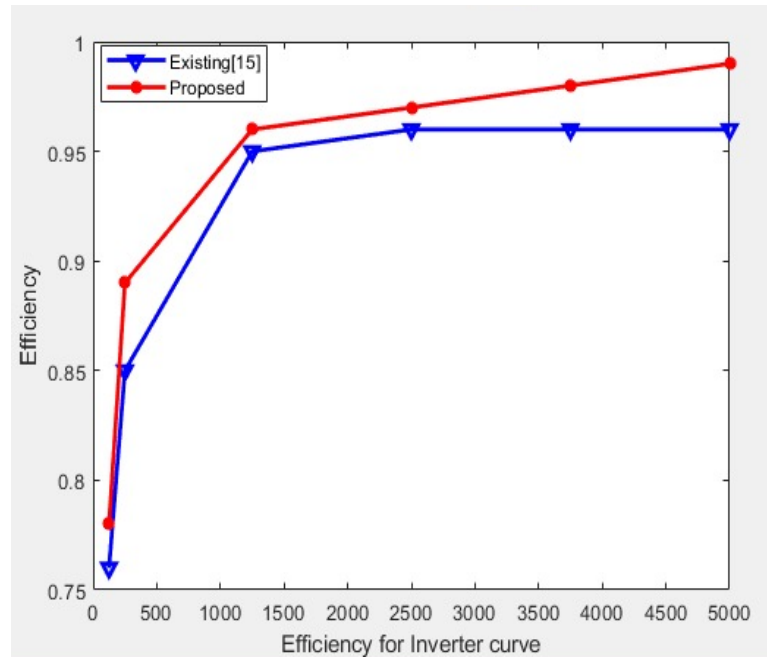

Figure 7 performance analysis of efficiency for inverter curve.

Figure 7 is the comparative analysis of inverter efficiency curve in terms of both existing and proposed system. The proposed technique offers better efficiency than existing technique.

Thus, the use of stochastic MFO aids in offering optimized duty cycle at which the optimal range of duty cycle is taken automatically for pulse generation. This aids in better performance by eradicating the distortion rate of grid current efficiently on comparing the existing techniques. Thus, the system is considered to be better than traditional ones.

\section{CONCLUSION}

This paper offers an effective scheme based on the Stochastic Moth Flame Optimization with fly back converter with H6 inverter introduction for the elimination of THD. An approach of soft-switching is attained by means of permitting negative current from the grid via the switches kept in the transformer's side. So, for optimizing reactive current Stochastic Moth Flame Optimization based MPPT was introduced for. This was being fed to switches in the flyback converter with H6 Inverter. From which the rate of THD was eliminated or reduced. Thus, for the dynamic system application the proposed method is employed. Finally, the power was stored in the grid side. The performance results authenticate the proposed system effectiveness that reduces THD successfully. 


\section{REFERENCES}

[1] A. Jendoubi, F. Tlili, F. J. M. Bacha, and C. i. Simulation, "Sliding mode control for a grid connected PV-system using interpolation polynomial MPPT approach," vol. 167, pp. 202218, 2020.

[2] A. Q. Al-Shetwi, M. Hannan, K. P. Jern, A. A. Alkahtani, and A. J. E. PG Abas, "Power Quality Assessment of Grid-Connected PV System in Compliance with the Recent Integration Requirements," vol. 9, p. 366, 2020.

[3] M. Mao, L. Zhang, H. Huang, B. Chong, and L. J. J. o. C. P. Zhou, "Maximum power exploitation for grid-connected PV system under fast-varying solar irradiation levels with modified salp swarm algorithm," vol. 268, p. 122158, 2020.

[4] M. S. Bouakkaz, A. Boukadoum, O. Boudebbouz, N. Fergani, N. Boutasseta, I. Attoui, et al., "Dynamic performance evaluation and improvement of PV energy generation systems using Moth Flame Optimization with combined fractional order PID and sliding mode controller," vol. 199, pp. 411-424, 2020.

[5] A. Fathy, H. Rezk, and D. J. S. E. Yousri, "A robust global MPPT to mitigate partial shading of triple-junction solar cell-based system using manta ray foraging optimization algorithm," vol. 207, pp. 305-316, 2020.

[6] S. A. Nowdeh, M. J. H. Moghaddam, S. Nasri, A. Y. Abdelaziz, M. Ghanbari, and I. Faraji, "A New Hybrid Moth Flame Optimizer-Perturb and Observe Method for Maximum Power Point Tracking in Photovoltaic Energy System," in Modern Maximum Power Point Tracking Techniques for Photovoltaic Energy Systems, ed: Springer, 2020, pp. 401-420.

[7] B. Yang, T. Zhu, J. Wang, H. Shu, T. Yu, X. Zhang, et al., "Comprehensive overview of maximum power point tracking algorithms of PV systems under partial shading condition," p. 121983, 2020.

[8] H. M. Sultan, A. S. Menesy, S. Kamel, A. Korashy, S. Almohaimeed, and M. J. A. E. J. Abdel-Akher, "An improved artificial ecosystem optimization algorithm for optimal configuration of a hybrid PV/WT/FC energy system," 2020.

[9] W. M. Zhang, Y. X. J. I. J. o. S. Zhang, and P. Modelling, "A novel method of reactive voltage optimisation for photovoltaic systems," vol. 15, pp. 145-154, 2020.

[10] A. M. Howlader, S. Sadoyama, L. R. Roose, and Y. Chen, "Active power control to mitigate voltage and frequency deviations for the smart grid using smart PV inverters," Applied Energy, vol. 258, p. 114000, 2020.

[11] M. Forcan and M. Maksimović, "Cloud-fog-based approach for smart grid monitoring," Simulation Modelling Practice and Theory, vol. 101, p. 101988, 2020.

[12] B. Taghezouit, F. Harrou, Y. Sun, A. H. Arab, and C. Larbes, "Multivariate statistical monitoring of photovoltaic plant operation," Energy Conversion and Management, vol. 205, p. 112317, 2020.

[13] S. Arab Ansari, J. S. Moghani, M. J. I. J. o. C. T. Mohammadi, and Applications, "Analysis and implementation of a new zero current switching flyback inverter," vol. 47, pp. 103$132,2019$.

[14] C. Chen, J. Li, S. Gao, and Y. Ma, "Design of Staggered Fly-back Single-phase Photovoltaic Grid-connected Micro-inverters," in 2019 IEEE 3rd Information Technology, 
Networking, Electronic and Automation Control Conference (ITNEC), 2019, pp. 17931798.

[15] J. Wang, S. Gao, Y. Sun, Z. Ji, L. Cheng, L. Li, et al., "Single Phase Bidirectional H6 Rectifier/Inverter," IEEE Transactions on Power Electronics, vol. 34, pp. 10710-10719, 2019. 